\title{
A new version of Jensen's inequality and related results
}

\author{
Gholamreza Zabandan ${ }^{1}$ and Adem Kılıçman²*
}

\section{"Correspondence:}

akilicman@putra.upm.edu.my

${ }^{2}$ Department of Mathematics and Institute of Mathematical Research, Universiti Putra Malaysia (UPM), Serdang, Selangor 43400 UPM,

Malaysia

Full list of author information is available at the end of the article

\section{Abstract}

In this paper we expand Jensen's inequality to two-variable convex functions and find the lower bound of the Hermite-Hadamard inequality for a convex function on the bounded area from the plane.

\section{Introduction}

Let $\mu$ be a positive measure on $X$ such that $\mu(X)=1$. If $f$ is a real-valued function in $L^{1}(\mu)$, $a<f(x)<b$ for all $x \in X$ and $\varphi$ is convex on $(a, b)$, then

$$
\varphi\left(\int_{X} f d \mu\right) \leq \int_{X}(\varphi \circ f) d \mu .
$$

The inequality (1) is known as Jensen's inequality [1].

In recent years, there have been many extensions, refinements and similar results of the inequality (1). Recall that the function $F: \Delta=[a, b] \times[c, d] \rightarrow \mathbb{R}$ is convex on $\Delta$ if

$$
F(\lambda x+(1-\lambda) z, \lambda y+(1-\lambda) w) \leq \lambda F(x, y)+(1-\lambda) F(z, w)
$$

holds for all $(x, y),(z, w) \in \Delta$ and $\lambda \in[0,1]$. A function $F: \Delta \rightarrow \mathbb{R}$ is called co-ordinated convex on $\Delta$ if the partial functions $F_{y}:[a, b] \rightarrow \mathbb{R}, F_{y}(u)=F(u, y)$ and $F_{x}:[c, d] \rightarrow \mathbb{R}$, $F_{x}(v)=F(x, v)$ are convex for all $x \in[a, b]$ and $y \in[c, d]$. Note that every convex function $F: \Delta \rightarrow \mathbb{R}$ is co-ordinated convex, but the converse is not generally true; see [2]. Also note that if $F$ is a convex function on $\mathbb{R}^{2}$ and $g, h$ are real-valued functions such that $D_{g}=D_{h}=\mathbb{R}$, then $f(t)=F(g(t), h(t))$ may be not convex on $\mathbb{R}$.

In this paper under suitable conditions, we expand Jensen's inequality to two-variable convex functions and deduce some further important inequalities. Finally, we find a lower bound for the integral

$$
\frac{1}{\int_{a}^{b}(g(x)-h(x)) d x} \int_{a}^{b} \int_{h(x)}^{g(x)} F(x, y) d y d x,
$$

where $F$ is convex on the convex bounded area by $y=g(x), y=h(x)$ and $x=a, x=b$.

\section{照 Springer}

๑) 2012 Zabandan and Kılıçman; licensee Springer. This is an Open Access article distributed under the terms of the Creative Commons Attribution License (http://creativecommons.org/licenses/by/2.0), which permits unrestricted use, distribution, and reproduction in any medium, provided the original work is properly cited. 


\section{Main results}

Theorem 1 Let $p$ be a non-negative continuous function on $[a, b]$ such that $\int_{a}^{b} p(x) d x>0$. If $g$ and h are real-valued continuous functions on $[a, b]$ and

$$
m_{1} \leq g(x) \leq M_{1}, \quad m_{2} \leq h(x) \leq M_{2}
$$

for all $x \in[a, b]$, and $F$ is convex on

$$
\Delta=\left[m_{1}, M_{1}\right] \times\left[m_{2}, M_{2}\right],
$$

then

$$
F\left(\frac{\int_{a}^{b} g(t) p(t) d t}{\int_{a}^{b} p(t) d t}, \frac{\int_{a}^{b} h(t) p(t) d t}{\int_{a}^{b} p(t) d t}\right) \leq \frac{\int_{a}^{b} F(g(t), h(t)) p(t) d t}{\int_{a}^{b} p(t) d t}
$$

and

$$
F\left(\frac{\int_{a}^{b} g(t) d t}{b-a}, \frac{\int_{a}^{b} h(t) d t}{b-a}\right) \leq \frac{1}{b-a} \int_{a}^{b} F(g(t), h(t)) d t .
$$

The inequalities hold in reversed order iff is concave on $\Delta$.

Proof Denote

$$
\alpha(x)=\frac{\int_{a}^{x} g(t) p(t) d t}{\int_{a}^{x} p(t) d t}
$$

and

$$
\beta(x)=\frac{\int_{a}^{x} h(t) p(t) d t}{\int_{a}^{x} p(t) d t} .
$$

Then by L'Hospital's rule, we have $\lim _{x \rightarrow a} \alpha(x)=g(a)$ and $\lim _{x \rightarrow a} \beta(x)=h(a)$. So, $\alpha$ and $\beta$ are continuous on $[a, b]$. Denote

$$
H(x)=F(\alpha(x), \beta(x))-\frac{\int_{a}^{x} F(g(t), h(t)) p(t) d t}{\int_{a}^{x} p(t) d t} .
$$

We will show that $H(b) \leq 0$. We have

$$
\begin{aligned}
H^{\prime}(x)= & \frac{\partial F(\alpha(x), \beta(x))}{\partial \alpha} \alpha^{\prime}(x)+\frac{\partial F(\alpha(x), \beta(x))}{\partial \beta} \beta^{\prime}(x) \\
& -\frac{F(g(x), h(x)) p(x)}{\int_{a}^{x} p(t) d t}+p(x) \frac{\int_{a}^{x} F(g(t), h(t)) p(t) d t}{\left(\int_{a}^{x} p(t) d t\right)^{2}} .
\end{aligned}
$$

By the convexity of $F$, we obtain

$$
\begin{aligned}
F(g(x), h(x))-F(\alpha(x), \beta(x)) \geq & \frac{\partial F(\alpha(x), \beta(x))}{\partial \alpha}(g(x)-\alpha(x)) \\
& +\frac{\partial F(\alpha(x), \beta(x))}{\partial \beta}(h(x)-\beta(x)) .
\end{aligned}
$$


So, we get

$$
\begin{aligned}
H^{\prime}(x) \leq & \frac{\partial(\alpha(x), \beta(x))}{\partial \alpha} \alpha^{\prime}(x)+\frac{\partial(\alpha(x), \beta(x))}{\partial \beta} \beta^{\prime}(x) \\
& -\frac{p(x)}{\int_{a}^{x} p(t) d t}\left[F(\alpha(x), \beta(x))+\frac{\partial(\alpha(x), \beta(x))}{\partial \alpha}(g(x)-\alpha(x))\right. \\
& \left.+\frac{\partial F(\alpha(x), \beta(x))}{\partial \beta}(h(x)-\beta(x))\right]+p(x) \frac{\int_{a}^{x} F(g(t), h(t)) p(t) d t}{\left(\int_{a}^{x} p(t) d t\right)^{2}} .
\end{aligned}
$$

Hence,

$$
\begin{aligned}
H^{\prime}(x) \leq & \frac{\partial(\alpha(x), \beta(x))}{\partial \alpha}\left[\alpha^{\prime}(x)-\frac{p(x)}{\int_{a}^{x} p(t) d t}(g(x)-\alpha(x))\right] \\
& +\frac{\partial(\alpha(x), \beta(x))}{\partial \beta}\left[\beta^{\prime}(x)-\frac{p(x)}{\int_{a}^{x} p(t) d t}(h(x)-\beta(x))\right] \\
& -\frac{p(x) F(\alpha(x), \beta(x))}{\int_{a}^{x} p(t) d t}+p(x) \frac{\int_{a}^{x} F(g(t), h(t)) g(t) d t}{\left(\int_{a}^{x} p(t) d t\right)^{2}} .
\end{aligned}
$$

By easy calculation, we see that

$$
\alpha^{\prime}(x)-\frac{p(x)}{\int_{a}^{x} p(t) d t}(g(x)-\alpha(x))=\beta^{\prime}(x)-\frac{p(x)}{\int_{a}^{x} p(t) d t}(h(x)-\beta(x))=0 .
$$

Therefore,

$$
H^{\prime}(x) \leq-\frac{p(x)}{\int_{a}^{x} p(t) d t}\left[F(\alpha(x), \beta(x))-\frac{\int_{a}^{x} F(g(t), h(t)) p(t) d t}{\int_{a}^{x} p(t) d t}\right]=-\frac{p(x)}{\int_{a}^{x} P(t) d t} H(x) .
$$

Thus,

$$
\left(\int_{a}^{x} p(t) d t\right) H^{\prime}(x)+p(x) H(x) \leq 0 \Rightarrow\left[\left(\int_{a}^{x} p(t) d t\right) H(x)\right]^{\prime} \leq 0 .
$$

So,

$$
\left(\int_{a}^{b} p(t) d t\right) H(b) \leq 0 \quad \Rightarrow \quad H(b) \leq 0 .
$$

The proof is complete. For the proof of (3), set $p(x)=1$.

Note the inequalities (2) and (3) are sharp because $F(x, y)=1$.

Corollary 1 Let $g$ and $h$ be real-valued continuous functions. Then we have

(i) for $\frac{1}{p}+\frac{1}{q}=1, p, q>1$,

$$
\int_{a}^{b}|g(t)||h(t)| d t \leq\left(\int_{a}^{b}|g(t)|^{p} d t\right)^{\frac{1}{p}}\left(\int_{a}^{b}|h(t)|^{q} d t\right)^{\frac{1}{q}} \quad \text { Holder's inequality, }
$$


Zabandan and Kılıçman Journal of Inequalities and Applications 2012, 0:238

Page 4 of 7

http://www.journalofinequalitiesandapplications.com/content/0/1/238

(ii) for $p \geq 1$,

$$
\begin{aligned}
& \left(\int_{a}^{b}|g(t)+h(t)|^{\frac{1}{p}} d t\right)^{p} \\
& \geq\left(\int_{a}^{b}|g(t)|^{\frac{1}{p}} d t+\int_{a}^{b}|h(t)|^{\frac{1}{p}} d t\right)^{p} \quad \text { reverse Minkowski's inequality, }
\end{aligned}
$$

(iii) for $p \geq 1$,

$$
\begin{aligned}
\left(\int_{a}^{b}|g(t)+h(t)|^{p} d t\right)^{\frac{1}{p}} \leq & \left(\int_{a}^{b}|g(t)|^{p} d t\right)^{\frac{1}{p}} \\
& +\left(\int_{a}^{b}|h(t)|^{p} d t\right)^{\frac{1}{p}} \quad \text { Minkowski's inequality, }
\end{aligned}
$$

(iv)

$$
\ln \left(e^{\frac{1}{b-a} \int_{a}^{b} g(t) d t}+e^{\frac{1}{b-a} \int_{a}^{b} h(t) d t}\right) \leq \frac{1}{b-a} \int_{a}^{b} \ln \left(e^{g(t)}+e^{h(t)}\right) d t .
$$

Proof

(i) The function

$$
F(x, y)=|x|^{\frac{1}{p}}|y|^{\frac{1}{q}} \quad\left(\frac{1}{p}+\frac{1}{q}=1\right),
$$

is concave, so by the inequality (3), we have

$$
\frac{\left(\int_{a}^{b}|g(t)| d t\right)^{\frac{1}{p}}}{(b-a)^{\frac{1}{p}}} \times \frac{\left(\int_{a}^{b}|h(t)| d t\right)^{\frac{1}{q}}}{(b-a)^{\frac{1}{q}}} \geq \frac{\int_{a}^{b}|g(t)|^{\frac{1}{p}}|h(t)|^{\frac{1}{q}} d t}{b-a} .
$$

Hence,

$$
\int_{a}^{b}|g(t)|^{\frac{1}{p}}|h(t)|^{\frac{1}{q}} d t \leq\left(\int_{a}^{b}|g(t)| d t\right)^{\frac{1}{p}}\left(\int_{a}^{b}|h(t)| d t\right)^{\frac{1}{q}} .
$$

Now, set $|g(t)| \rightarrow|g(t)|^{p}$ and $|h(t)| \rightarrow|h(t)|^{q}$. We obtain

$$
\int_{a}^{b}|g(t)||h(t)| d t \leq\left(\int_{a}^{b}|g(t)|^{p} d t\right)^{\frac{1}{p}}+\left(\int_{a}^{b}|h(t)|^{q} d t\right)^{\frac{1}{q}} .
$$

(ii) The function $F(x, y)=\left(|x|^{p}+|y|^{p}\right)^{\frac{1}{p}}$ is convex for $p \geq 1$ and is concave for $p<1$. So, by the inequality (3), we have

$$
\left[\frac{\left(\int_{a}^{b}|g(t)| d t\right)^{p}}{(b-a)^{p}}+\frac{\left(\int_{a}^{b}|h(t)| d t\right)^{p}}{(b-a)^{p}}\right]^{\frac{1}{p}} \leq \frac{\int_{a}^{b}\left(|g(t)|^{p}+|h(t)|^{p}\right)^{\frac{1}{p}} d t}{b-a}
$$

so

$$
\int_{a}^{b}\left(|g(t)|^{p}+|h(t)|^{p}\right)^{\frac{1}{p}} d t \geq\left[\left(\int_{a}^{b}|g(t)| d t\right)^{p}+\left(\int_{a}^{b}|h(t)| d t\right)^{p}\right]^{\frac{1}{p}} .
$$


Now, set $|g(t)| \rightarrow|g(t)|^{\frac{1}{p}}$ and $|h(t)| \rightarrow|h(t)|^{\frac{1}{p}}$. We get

$$
\int_{a}^{b}(|g(t)|+|h(t)|)^{\frac{1}{p}} d t \geq\left[\left(\int_{a}^{b}|g(t)|^{\frac{1}{p}} d t\right)^{p}+\left(\int_{a}^{b}|h(t)|^{\frac{1}{p}} d t\right)^{p}\right]^{\frac{1}{p}}
$$

So,

$$
\left(\int_{a}^{b}(|g(t)|+|h(t)|)^{\frac{1}{p}} d t\right)^{p} \geq\left(\int_{a}^{b}|g(t)|^{\frac{1}{p}} d t\right)^{p}+\left(\int_{a}^{b}|h(t)|^{\frac{1}{p}} d t\right)^{p} .
$$

The proof of (iii) is similar to that of (ii) and can be omitted. For the proof of (iv), note $f(x, y)=\ln \left(e^{x}+e^{y}\right)$ is convex on $\mathbb{R}^{2}$. Now, apply the inequality (3).

Remark 1 By similar assumptions, we can prove Theorem 1 for an $n$-variable convex function $F$ on $\mathbb{R}^{n}$ and obtain the inequality

$$
F\left(\frac{\int_{a}^{b} g_{1}(t) d t}{b-a}, \ldots, \frac{\int_{a}^{b} g_{n}(t) d t}{b-a}\right) \leq \frac{1}{b-a} \int_{a}^{b} F\left(g_{1}(t), \ldots, g_{n}(t)\right) d t
$$

In particular, we can obtain a similar inequality for Holder and Minkowski inequalities. For example, by the concavity of

$$
F\left(t_{1}, t_{2}, \ldots, t_{n}\right)=\prod_{i=1}^{n}\left|t_{i}\right|^{\frac{1}{p_{i}}} \quad\left(\sum_{i=1}^{n} \frac{1}{p i}=1\right)
$$

we can get the inequality

$$
\int_{a}^{b}\left(\prod_{i=1}^{n}\left|g_{i}\right|\right) d t \leq \prod_{i=1}^{n}\left(\int_{a}^{b}\left|g_{i}\right|^{p_{i}}\right)^{\frac{1}{p_{i}}}
$$

\section{Hermite-Hadamard inequality}

Let $f:[a, b] \rightarrow \mathbb{R}$ be a convex function, then the following inequality is known as the Hermite-Hadamard inequality [3] and [4]:

$$
f\left(\frac{a+b}{2}\right) \leq \frac{1}{b-a} \int_{a}^{b} f(x) d x \leq \frac{f(a)+f(b)}{2}
$$

In [5], Dragomir established the following similar inequality (4) for convex functions on the co-ordinates on a rectangle from the plane $\mathbb{R}^{2}$.

Theorem 2 Suppose $f: \Delta=[a, b] \times[c, d] \rightarrow \mathbb{R}$ is a convex function on the co-ordinates on $\triangle$. Then one has the inequalities

$$
\begin{aligned}
f\left(\frac{a+b}{2}, \frac{c+d}{2}\right) & \leq \frac{1}{(b-a)(d-c)} \int_{a}^{b} \int_{c}^{d} f(x, y) d y d x \\
& \leq \frac{f(a, c)+f(a, d)+f(b, c)+f(b, d)}{4}
\end{aligned}
$$


Also Dragomir investigated the Hermite-Hadamard inequality on the disk [6] and [7]. In [8], Matejička proved the left-hand side of the Hermite-Hadamard inequality of several variables for a convex function on certain convex compact sets. In the following theorem, we prove the left-hand side of the Hermite-Hadamard inequality in another way and as a result of Theorem 2 .

Theorem 3 Let $\triangle$ be a bounded area by a convex function $h$ and a concave function $g$ on $[a, b]$ such that for any $x \in[a, b], g(x) \geq h(x)$. Also, let $F$ be a two-variable convex function on $\triangle$. Then one has the inequality

$$
F\left(\frac{\int_{a}^{b} x(g(x)-h(x)) d x}{\int_{a}^{b}(g(x)-h(x)) d x}, \frac{\frac{1}{2} \int_{a}^{b}\left(g^{2}(x)-h^{2}(x)\right) d x}{\int_{a}^{b}(g(x)-h(x)) d x}\right) \leq \frac{\int_{a}^{b} \int_{h(x)}^{g(x)} F(x, y) d y d x}{\int_{a}^{b}(g(x)-h(x)) d x} .
$$

Proof Since $F$ is convex on $\triangle$, hence $f$ is co-ordinated convex on $\triangle$. So, $F_{x}:[h(x), g(x)] \rightarrow$ $\mathbb{R}, F_{x}(y)=F(x, y)$ is convex on $[h(x), g(x)]$ for all $x \in[a, b]$. By the left-hand side of the Hermite-Hadamard inequality (4), we have

$$
(g(x)-h(x)) F\left(x, \frac{g(x)+h(x)}{2}\right) \leq \int_{h(x)}^{g(x)} F(x, y) d y .
$$

Integrating this inequality on $[a, b]$, we obtain

$$
\int_{a}^{b}(g(x)-h(x)) F\left(x, \frac{g(x)+h(x)}{2}\right) d x \leq \int_{a}^{b} \int_{h(x)}^{g(x)} F(x, y) d y d x .
$$

So,

$$
\frac{\int_{a}^{b}(g(x)-h(x)) F\left(x, \frac{g(x)+h(x)}{2}\right) d x}{\int_{a}^{b}(g(x)-h(x)) d x} \leq \frac{1}{\int_{a}^{b}(g(x)-h(x)) d x} \int_{a}^{b} \int_{h(x)}^{g(x)} F(x, y) d y d x .
$$

Now, let $p(x)=g(x)-h(x)$. By the inequality (2), we get

$$
\begin{aligned}
F\left(\frac{\int_{a}^{b} x(g(x)-h(x)) d x}{\int_{a}^{b}(g(x)-h(x)) d x}, \frac{\frac{1}{2} \int_{a}^{b}\left(g^{2}(x)-h^{2}(x)\right) d x}{\int_{a}^{b}(g(x)-h(x)) d x}\right) & \leq \frac{\int_{a}^{b}(g(x)-h(x)) F\left(x, \frac{g(x)+h(x)}{2}\right) d x}{\int_{a}^{b}(g(x)-h(x)) d x} \\
& \leq \frac{\int_{a}^{b}(g(x)-h(x)) F\left(x, \frac{g(x)+h(x)}{2}\right) d x}{\int_{a}^{b}(g(x)-h(x)) d x} .
\end{aligned}
$$

The proof is complete.

\section{Author details}

${ }^{1}$ Department of Mathematics, Faculty of Mathematical Sciences and Computer, Tarbiat Moallem University, 599 Taleghani Avenue, Tehran, 15618, Iran. ${ }^{2}$ Department of Mathematics and Institute of Mathematical Research, Universiti Putra Malaysia (UPM), Serdang, Selangor 43400 UPM, Malaysia. 
References

1. Rudin, W: Real and Complex Analysis. McGraw-Hill, New York (1974)

2. Dragomir, SS: On Hadamard's inequality for the convex mappings defined on a ball in the space and application. Math. Inequal. Appl. 3, 177-187 (2000)

3. Mitrinovic, DS, Lackoric, JB: Hermite and convexity. Aequ. Math. 28, 229-232 (1985)

4. Zabandan, G: A new refinement of the Hermite-Hadamard inequality for convex functions. JIPAM. J. Inequal. Pure Appl. Math. 10(2), Art. 45 (2009)

5. Dragomir, SS: On the Hadamard's inequality for convex functions on the co-ordinates in a rectangle from the plane. Taiwan. J. Math. 5, 775-788 (2001)

6. Dragomir, SS: On Hadamard's inequality on a disk. J. Inequal. Pure Appl. Math. 1, Article 2 (2000)

7. Dragomir, SS, Pearce, CEM: Selected Topics on Hermite-Hadamard Inequalities. RGMIA Monographs, Victoria University (2000)

8. Matejíčka, L: Elementary proof of the left multidimensional Hermite-Hadamard inequality on certain convex sets. J. Math. Inequal. 4(2), 259-270 (2010)

doi:10.1186/1029-242X-2012-238

Cite this article as: Zabandan and Kılıçman: A new version of Jensen's inequality and related results. Journal of Inequalities and Applications 2012 0:238.

\section{Submit your manuscript to a SpringerOpen ${ }^{\circ}$ journal and benefit from:}

- Convenient online submission

- Rigorous peer review

- Immediate publication on acceptance

Open access: articles freely available online

- High visibility within the field

- Retaining the copyright to your article 